\title{
Ultrasound therapy for recurrent noninfective olecranon bursitis: A case report
}

\author{
S. Aswinprakash, D. Jagadeesh, R. Arulmoli, Yuvaraj Maria Francis ${ }^{1}$, Kaviarasu Mahalingam², \\ Robert Francis Stanley ${ }^{3}$, Diwakar Aiyaloo ${ }^{4}$
}

Anatomy Unit, Faculty of Medicine, AIMST University, ${ }^{2}$ Physiotherapy Unit, Faculty of Allied Health Professions, AIMST University, ${ }^{3}$ Physiology Unit, Faculty of Medicine, AIMST University, ${ }^{4}$ Biochemistry Unit, Faculty of Medicine, AIMST University, Bedong, Kedah, Malaysia, ${ }^{1}$ Department of Anatomy, Saveetha Medical College, Thandalam, Chennai, India

Abstract Olecranon bursa is the commonly involved structure of the elbow joint in trauma of any mechanical cause or infections due to its subcutaneous position. The overall incidence may vary, but it is more common in males between 30 and 60 years. Various pharmaceutical and physiotherapeutic treatment options are available based on septic or nonseptic conditions. Mostly antibiotics and nonsteroidal anti-inflammatory drugs are widely used by physicians, whereas electrotherapy modalities are the choice of treatment by physiotherapists. Among which, ultrasound (US) therapy is found to be more convenient and effective in treating bursitis. As of the recent evidence available, US therapy is highly effective in treating olecranon bursitis.

Keywords: Bursitis, elbow, olecranon, orthopedics, trauma, ultrasound therapy

Address for correspondence: Mr. S. Aswinprakash, Lecturer, Anatomy Unit, Faculty of Medicine, AIMST University, Semeling Bedong, Kedah, Malaysia 01800. E-mail: aswin.arien17@gmail.com

\section{INTRODUCTION}

Olecranon bursa is a thin fluid-filled sac located at the tip of the elbow joint. ${ }^{[-3]}$ This superficial bursa usually develops between 7 and 10 years of age. ${ }^{[4]}$ The floor of the olecranon bursa lies on the triceps tendon and olecranon, and the roof is loosely connected to the overlying skin of the elbow. ${ }^{[5]}$ The function is to prevent the friction between the olecranon process and the subcutaneous tissues during both flexion and extension of the elbow joint. ${ }^{[6]}$ The location of this bursa itself makes it more prone to any kind of trauma or infection. The term olecranon bursitis is represented using various terms such as swollen elbow, student's elbow, and Baker's elbow. ${ }^{[7]}$

The clinical features are characterized by pain, swelling, and inflammation at the point of the elbow. The overall incidence

\begin{tabular}{|l|l|}
\hline \multicolumn{2}{|c|}{ Access this article online } \\
\hline Quick Response Code: & Website: \\
\hline & www.jpionline.org \\
\cline { 2 - 2 } & DOI: \\
\hline
\end{tabular}

is not certain; it typically affects men between the ages of 30 and 60 years. ${ }^{[8,9]}$ Nearly two-thirds of cases were noninfectious types, ${ }^{[10]}$ and usually, it is a sterile inflammation that develops secondary to any acute, occupational, or recreational trauma, crystal deposition like gout, and systemic autoimmune diseases such as rheumatoid arthritis and systemic lupus erythematosus. Most cases of nonseptic bursitis are posttraumatic or due to overuse injuries (repeated minor trauma due to sports) or direct trauma. This causes bleeding within the bursa or release of inflammatory substances.

In the conservative management, anti-inflammatory and antibiotic medications were prescribed based on the severity. Adjunct to that, various modalities such as

This is an open access journal, and articles are distributed under the terms of the Creative Commons Attribution-NonCommercial-ShareAlike 4.0 License, which allows others to remix, tweak, and build upon the work non-commercially, as long as appropriate credit is given and the new creations are licensed under the identical terms.

For reprints contact: reprints@medknow.com

How to cite this article: Aswinprakash S, Jagadeesh D, Arulmoli R, Francis YM, Mahalingam K, Stanley RF, et al. Ultrasound therapy for recurrent noninfective olecranon bursitis: A case report. Int J Pharma Investig 2018;8:205-9. 
cryotherapy, taping techniques, compression, and optimal elbow positioning and padding have been done to promote the healing. Aspiration, corticosteroid injection, and surgical drainage and excision are only considered in severe and recurrent cases.

Ultrasound (US) therapy is one of the electrotherapy modalities used in physiotherapy for treating inflammatory conditions, ${ }^{[11]}$ in which electrical energy is converted into acoustic energy by means of deformation of piezoelectric crystal present within the transducer. Therapeutic US administered over the tissues will be having thermal and nonthermal effects. Thermal effects aid in pain relief, whereas nonthermal effects enhance cell repair. ${ }^{[12]}$ The physiological effects had been proved and commonly used for the soft-tissue injuries and associated rheumatic complaints. ${ }^{[13]}$ This case report presents a unique treatment for recurrent noninfective olecranon bursitis using US therapy. After the failure of conservative management, a patient with recurrent nonseptic olecranon bursitis was treated with US therapy and completely recovered. This therapy provided a safe method without creating any discomfort for the patient. It could be considered as an attractive treatment option for chronic nonseptic olecranon bursitis.

\section{CASE REPORT}

A 22-year-old healthy man, who is a regular basket ballplayer, presented the complaints with left posterior elbow swelling, pain, and discomfort for nearly 6 months [Figure 1]. He had a history of elbow trauma by hitting the point of the elbow directly against the concrete floor of the basketball court, as a result of falling during sports activity. The pain tends to increase during rest at the affected elbow. He continued to play basketball and is able to do functional activities with a lesser pain and discomfort. However, he denied any paresthesia around the elbow or weakness.

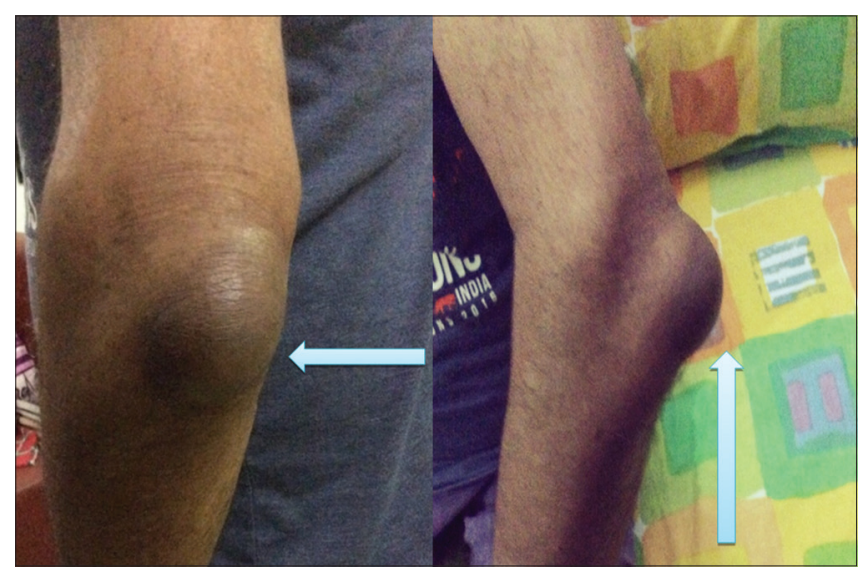

Figure 1: Olecranon bursitis of the left elbow
He was prescribed with nonsteroidal anti-inflammatory drugs (NSAIDs) and analgesics by an orthopedic surgeon who attended him initially, with a follow-up of one month and no further significant improvement, aspirations and two corticosteroid injections were administered into his affected left olecranon bursa; adhesive bandage was applied over the point of the elbow without any immobilization of the joint. This made a temporary resolution of the condition, but the swelling appeared in the posterior elbow after 2 weeks.

Laboratory investigations were done, and the fluid culture turned to be negative and all markers were in the normal range. The patient refused for any further invasive surgical procedures and referred for the physiotherapy management to New Life Hospital, Chennai. During the assessment, the physical examination of the left elbow point showed an average of $5.5 \mathrm{~cm}$ and bounded mass over the olecranon process without any tenderness, redness, or warmth. Goniometric active and passive range of elbow flexion and extension in the affected elbow was normal $\left(0^{\circ}-120^{\circ}\right)$. The movements at the radioulnar joint (supination and pronation) were normal. There was no abnormal neurological involvement. No spasm or wasting of the muscles was observed.

The radiographical findings revealed the soft-tissue enlargement over the olecranon process without any kind of fracture or abnormal bony growth. Based on the overall clinical status and the fixed condition, it was diagnosed as recurrent noninfective olecranon bursitis. The patient expressed and preferred his interest in trying physiotherapy management using electrotherapy modality. The decision was made with US therapy and informed the patient about the procedure.

Chattanooga brand US machine with the transducer head size of $5 \mathrm{~cm}^{2}$ was used for treating this patient [Figure 2].

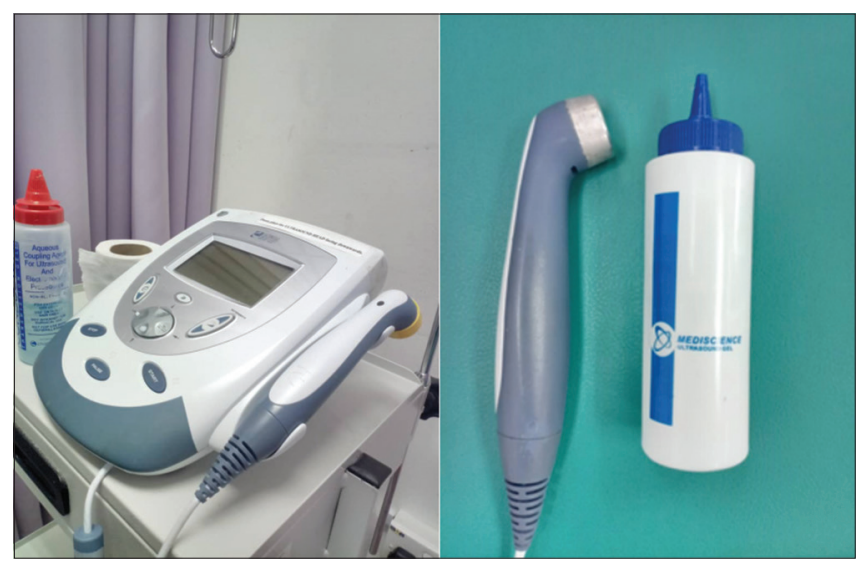

Figure 2: Therapeutic ultrasound with plugged in transducer and coupling media 
Effective radiated area was selected around the tip of the elbow at the site of swelling with their adjacent margins; after sterilizing the treatment area, coupling media were applied over the affected site and the transducer head. The transducer was moved in a circular and longitudinal pattern over the site of swelling and its boundaries. The frequency of $3 \mathrm{MHz}$ in continuous mode with a ratio of $1: 1$ and intensity of about $0.5-0.8 \mathrm{~W} / \mathrm{cm}^{2}$ was employed. The duration of the treatment was 5-10 min every other day. The patient attended 14 sittings for the therapy with progressive changes, and complete resolution was achieved grossly during the final stages of therapy. Posttreatment evaluations were done in a regular follow-up of 2 weeks, 1 month, 2 months, and 6 months. He continued to report with no recurrence, and physical examinations showed neither swelling nor discomfort around the point of the elbow. There was a complete pain-free range of motion of the elbow joint, and the patient was satisfied with the outcome.

\section{DISCUSSION}

Recurrent noninfective olecranon bursitis will be exasperation for the patients and the physicians who are managing the condition. Sometimes, there will be disability ranging that will alter the functional activities of daily living. The reason is because of increase in the inflammatory fluid within the bursa. The swelling around the point of the elbow is considered in a cosmetic point of view. Even though a wide variety of NSAIDs available and various invasive surgical interventions such as bursectomy as the choice of treatments the recurrence after all these measures will be a frustrating factor for the physician as well as the patient.

In a study of Stasinopoulos et al., US is one of the modalities used by those physiotherapists daily in their clinical practice. There are strong evidence that support US for producing positive effects on soft-tissue healing. ${ }^{[14]}$ Similar effects have been observed on animal studies as well. ${ }^{[15,16]}$ Scientifically, it has been proved that the effectiveness of US was based on its parameters: frequency, mode, intensity, duration of treatment, the way the transducer head was moved during the treatment, coupling medium, treatment intervals, and effective radiated area.

In a study of done by Draper and Prentice, US is a deep-heating modality. Acoustic microstreaming is defined as the physical forces of the sound waves that provide a driving force capable of displacing ions and small molecules. At a soft-tissue depth of $3 \mathrm{~cm}$, a 10-min therapy induces an increase of $0.8^{\circ} \mathrm{C}$, and $1-\mathrm{MHz}$ US has raised the temperature nearer to $4^{\circ} \mathrm{C}$ by $10 \mathrm{~min}$, whereas by $1 \mathrm{~cm}$ below the fat surface, a 4-minute warm whirlpool $\left(40.6^{\circ} \mathrm{C}\right)$ raised the temperature $1.1^{\circ} \mathrm{C}$; however, at this same depth, 3-MHz US raised the temperature $4^{\circ} \mathrm{C}$ in 4 min. Thermal effects of the US over the body tissues cause an increase in circulation, reduction of muscle spasm, and extensibility of collagen fibers. ${ }^{[17]}$ In a study of done by Dyson et al., nonthermal effects of the US are the cavitation, in which the microstreaming causes the increase in flow of surrounding fluid. This kind of effect will reduce the swelling. Nonthermal effects occur when pulsed US is applied. Nonthermal effects are useful for decreasing edema and promoting cellular repair. The above-said changes are the result of the chemical, biologic, mechanical, and thermal effects of the sound waves. ${ }^{[18]}$

Physiological effects of US are considered to increase in collagen extensibility, increases the nerve conduction velocities, increases the metabolism of edema and exudates, decreases joint stiffness, increases pain threshold, decreases muscle spasm and releases histamine. The indications of US therapy are soft-tissue injuries, myositis ossificans, chronic connective tissue and joint dysfunction, nerve entrapments, osteoarthritis, plantar warts, periarthritis (nonseptic), chronic sprains and strains, tenosynovitis, muscle spasm, tendonitis, bursitis, and capsulitis. ${ }^{[19]}$

It was demonstrated that there is a direct relationship between the absorption of US and the amount of protein in the tissue, and when the concentration of protein increased, the absorption of US increased. In normal tissue, the absorption of US energy varies depending on the amount of protein in the tissue. ${ }^{[20]}$ Love and Kremkau et al. demonstrated that there is a direct relationship between the absorption of US and the amount of protein in the tissue, and when the concentration of protein increased, the absorption of US increased. In normal tissue, the absorption of US energy varies depending on the amount of protein in the tissue, they removed extracellular tissue structures such as collagen, fibrin, and elastin, and then placing only the cells in tissue culture media maintained at $37^{\circ} \mathrm{C}$, they could treat cells at therapeutic levels without significant increases in temperature $<0.5^{\circ} \mathrm{C}$ for $>10$ min. ${ }^{[21]}$

The application of US during the inflammatory, proliferative, and tissue repair stages is not only of value because it changes the normal sequence of events but also because it has the capacity to stimulate or enhance these normal events and thus increase the efficiency in the healing stages. ${ }^{[2]}$ During the inflammatory phase, US has a stimulating effect on the mast cells, platelets, white cells with phagocytic roles, and macrophages. ${ }^{[23]}$ The US application induces degranulation of mast cells, causing the release of arachidonic acid which itself is a precursor for the synthesis of prostaglandins and 
leukotriene which in turn act as inflammatory mediators. ${ }^{[2]}$ US has a stimulative effect during the proliferative phase and scar production through the fibroblasts, endothelial cells, and myofibroblasts. Therefore, US does not change the normal proliferative phase but maximizes its efficiency - producing the required scar tissue in an optimal fashion - and low-dose pulsed US increases protein synthesis, and several research groups have demonstrated enhanced fibroplasia and collagen synthesis. ${ }^{[25]}$ The therapeutic application of US influences over the remodeling of scar tissue by enhancing the appropriate orientation of the newly formed collagen fibers, increasing the viscoelastic property, and enhancing scar tissue mobilization. ${ }^{[26,27]}$

Regarding the effectiveness of US, Robertson and Baker concluded that there are little evidence that US was very effective than placebo for treating people with musculoskeletal conditions or for promoting soft-tissue healing. There are few studies deemed to have adequate methods examined a wide that range of patient problems, the dosages used in these studies varied considerably, often for no discernable reasons. ${ }^{[28]}$ Indeed, some of the studies from review could not draw a definitive conclusion because of insufficient evidence. Several reviews also reported disagreement and confusion about the most efficacious treatment parameters for US. Studies concerning the biophysical effects have been performed in vitro and have not been shown to have a clinical effect under therapeutic conditions. ${ }^{[2]}$ However, despite the confusion and disagreement among studies, reports from clinicians suggest that US remains a popular therapeutic modality, and many experienced and advanced practice clinicians continue to use US regularly for specific conditions. Few significant publications support the use of US in the connective tissue diseases such as osteoarthritis, Carpal tunnel syndrome, lateral epicondylitis, and myofascial pain syndrome. ${ }^{[30]}$ Even though the therapeutic effects of US over various soft-tissue inflammations were listed among the literature. There is no review in the survey which shows the treatment outcomes of US with respect to olecranon bursitis. In the present report, the efficiency of the US over recurrent noninfective olecranon bursitis was recorded.

\section{CONCLUSION}

In this case, a novel treatment for olecranon bursitis was explained. The recurrence of the bursal effusion was prevented by the routine ultrasonic therapy sessions. Although recurrent noninfective olecranon bursitis could be well managed by conservative or surgical management, US therapy may be beneficial as of the patients' choice of treatment. This modality could be prescribed for the acute and chronic soft-tissue pathologies similar to the above-mentioned condition.

\section{Declaration of patient consent}

The authors certify that they have obtained all appropriate patient consent forms. In the form the patient(s) has/have given his/her/their consent for his/her/their images and other clinical information to be reported in the journal. The patients understand that their names and initials will not be published and due efforts will be made to conceal their identity, but anonymity cannot be guaranteed.

Financial support and sponsorship

Nil.

\section{Conflicts of interest}

There are no conflicts of interest.

\section{REFERENCES}

1. Shell D, Perkins R, Cosgarea A. Septic olecranon bursitis: Recognition and treatment. J Am Board Fam Pract 1995;8:217-20.

2. Ho G Jr., Tice AD, Kaplan SR. Septic bursitis in the prepatellar and olecranon bursae: An analysis of 25 cases. Ann Intern Med 1978;89:21-7.

3. Reilly JP, Nicholas JA. The chronically inflamed bursa. Clin Sports Med 1987;6:345-70.

4. Chen J, Alk D, Eventov I, Wientroub S. Development of the olecranon bursa. An anatomic cadaver study. Acta Orthop Scand 1987;58:408-9.

5. Reilly D, Kamineni S. Olecranon bursitis. J Shoulder Elbow Surg 2016;25:158-67.

6. Blackwell JR, Hay BA, Bolt AM, Hay SM. Olecranon bursitis: A systematic overview. Shoulder Elbow 2014;6:182-90.

7. Eltorai AE, Eberson CP, Daniels AH, editors. Orthopedic Surgery Clerkship: A Quick Reference Guide for Senior Medical Students. Springer; 2017. p. 92-5.

8. Berkoff DJ, Sandbulte ZW, Stafford HC, Berkowitz JN. Fibrin glue for olecranon bursitis: A case report. Ther Adv Musculoskelet Dis 2016;8:28-30.

9. Stell IM. Septic and non-septic olecranon bursitis in the accident and emergency department - An approach to management. J Accid Emerg Med 1996;13:351-3.

10. Lockman L. Treating nonseptic olecranon bursitis: A 3-step technique. Can Fam Physician 2010;56:1157.

11. Thiruvasagar P. Effectiveness of ultrasound therapy in combination with manual therapy and shoulder exercises for sub acromial impingement syndrome. Int J Sci Res Publ 2013;3:8.

12. Speed CA. Therapeutic ultrasound in soft tissue lesions. Rheumatology (Oxford) 2001;40:1331-6.

13. Saber AA, Saber A. Therapeutic ultrasound: Physiological Role, clinical applications and precautions. J Surg 2017;5:61-9.

14. Stasinopoulos D, Cheimonidou AZ, Chatzidamianos T. Are there effective ultrasound parameters in the management of lateral elbow tendinopathy? A systematic review of the literature. Int J Phys Med Rehabil 2013;1:2-4.

15. Gan BS, Huys S, Sherebrin MH, Scilley CG. The effects of ultrasound treatment on flexor tendon healing in the chicken limb. J Hand Surg Br 1995;20:809-14.

16. da Cunha A, Parizotto NA, Vidal Bde C. The effect of therapeutic ultrasound on repair of the Achilles tendon (tendo calcaneus) of the rat. Ultrasound Med Biol 2001;27:1691-6. 
17. Draper DO, Prentice WE. Therapeutic ultrasound. In: Prentice WE, Quillen WS, Underwood F Prentice WE, Quillen WS, editors. Therapeutic Modalities in Rehabilitation. $4^{\text {th }}$ ed., Ch. 10. McGraw Hill Professional; 2017.

18. Dyson M. Mechanisms involved in therapeutic ultrasound. Physiotherapy1987;73:116-20.

19. Physiological Techniques, 3-Therapeutic Ultrasound. Ch. 4. New York Chiropractic College; 2003.

20. Stewart HG, Stratmeyer ME. An Overview of Ultrasound: Theory, Measurement, Medical Applications, and Biological Effects. Washington, DC: Department of Health, Education, and Welfare; DHEW Publication; 1982. p. 82-190.

21. Love LA, Kremkau FW. Intracellular temperature distribution produced by ultrasound. J Acoust Soc Am 1980;67:1045-50.

22. Watson T. Ultrasound in contemporary physiotherapy practice. Ultrasonics 2008;48:321-9.

23. Maxwell L. Therapeutic ultrasound: Its effects on the cellular and molecular mechanisms of inflammation and repair. Physiotherapy 1992;78:421-6

24. Leung MC, Ng GY, Yip KK. Effect of ultrasound on acute inflammation of transected medial collateral ligaments. Arch Phys Med Rehabil 2004;85:963-6.

25. Zhou S, Schmelz A, Seufferlein T, Li Y, Zhao J, Bachem MG, et al. Molecular mechanisms of low intensity pulsed ultrasound in human skin fibroblasts. J Biol Chem 2004;279:54463-9.

26. Nussbaum E. The influence of ultrasound on healing tissues. J Hand Ther 1998;11:140-7.

27. Maan ZN, Januszyk M, Rennert RC, Duscher D, Rodrigues M, Fujiwara $\mathrm{T}$, et al. Noncontact, low-frequency ultrasound therapy enhances neovascularization and wound healing in diabetic mice. Plast Reconstr Surg 2014;134:402e-11e.

28. Robertson VJ, Baker KG. A review of therapeutic ultrasound: Effectiveness studies. Phys Ther 2001;81:1339-50.

29. Page MJ, Green S, Mrocki MA, Surace SJ, Deitch J, McBain B, et al. Electrotherapy modalities for rotator cuff disease. Cochrane Database Syst Rev 2016;CD012225.

30. Daniel A. Martinez, Therapeutic Ultrasound: A Review of the Literature. Chiro Access; 01 October, 2010. Available from: http://www.chiroaccess. $\mathrm{com} /$ Articles/Therapeutic-Ultrasound-A-Review-of-the-Literature. aspx?id=0000210. [Last accessed on 2019 Mar 30]. 